\title{
Of germs and men
}

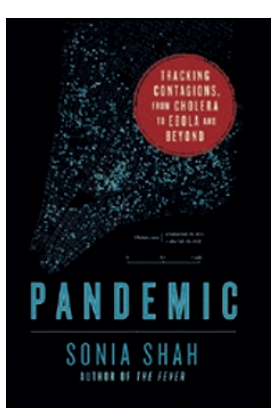

Pandemic: Tracking

Contagions, from

Cholera to Ebola

and Beyond

by Sonia Shah

SARAH CRICHTON

BOOKS: 2016. 258PP

US $\$ 26.00$

$\Lambda$ fter witnessing first-hand the media hunger for quick and scary Ebola tales, I was pleasantly surprised upon reading Sonia Shah's latest book, Pandemic. The distinguished science journalist takes us through the long, yet shrewdly articulated, journey that leads to human pandemics. Using Vibrio cholerae and contemporary pathogens such as Ebola as examples, she unpicks the social, political and, to some extent, biological conditions favouring the development of a pandemic. Half a decade of meticulous journalistic effort, travelling the world, meeting eminent researchers and the populations affected by pandemics have shaped her comprehensive perspective on the topic.

Shah first describes our role in promoting the jump of a pathogen from animals to humans. Human activities - such as colonization of a new land, deforestation or intensive livestock farming - lead to rapid changes in ecosystems, altering the opportunities for contact between animals and humans, and hence the risk of spillover of a pathogen into humans, as well as promoting evolution towards more transmissible strains. Once a pathogen successfully evolves to become transmissible between humans, it is likely to provoke a pandemic in our ever-growing and mobile human population. Cholera and Ebola are two such examples. Cholera emerged in humans in the eighteenth century in the newly colonized Sundarbans, rapidly spread globally by boat, and exploded in 1832 in a crowded New York City. Almost two centuries later, Ebola emerged in a deforested area of rural Guinea, propitious to spillover and in close proximity to large cities, perfect to catalyse the spread.

Shah explains how humans endeavour to develop cures and tools against contagion.
She touches upon the associated biological challenges, but focuses on other difficulties. She shows that the scientific community can sometimes hinder prevention efforts by dismissing new, outside-the-box theories. It was only about 40 years after John Snow's death that his theory (that cholera was water- and not air-borne) was finally widely accepted. I would argue that we, scientists and scientific editors, have the responsibility to ensure that scientific discoveries are judged on their soundness rather than their trendiness; I think Ebola has shown that this is not yet the case.

The success or failure of implementing technological solutions, Shah argues, also depends on political forces. For example, preventing cholera transmission is technically straightforward; simple water sanitation systems, already in place in Roman times, can dramatically reduce its toll. But Shah relates how in 1799, construction of waterworks in New York City was entrusted to a private company, which - in order to reduce costs - decided to use cheap poor materials and to take water from a filthy, but easily accessible, source. Shah explains that humans' "deeply rooted $[. .$.$] capacity for cooperative action$ failed" because immediate private benefits had been favoured over potential future common interests, and warns against the privatization of public goods. As detailed in the biting and captivating chapter entitled 'Corruption', our current global health management system suffers from the same drawbacks. It has failed at preserving its independence by relying largely on private funding, and has not managed to overcome divergences between local and global interests. Such political intricacies were decisive, Shah rightly argues, in the rapid expansion of the West African Ebola epidemic in 2013-2014.

Another key element potentially impairing human responses to pandemics is social pressure. Marginalization of a specific group, rightly or wrongly identified by the community as responsible for the contagion, can hinder effective prevention. Shah explains that doctors were often accused of killing patients in choleraaffected New York City, whilst immigrants were refused housing, increasing their risk of contracting the disease. And she shows that this remains a contemporary problem, reminding us that the Ebola outbreak in West Africa was particularly challenging to contain because of mistrust in governments and health providers.

So how can we prevent the next pandemic, be it caused by a new pathogen or an old one? Although Sonia Shah doesn't provide a magical answer, as might be suggested by the back cover, she gives the reader food for thought. She reminds us that we, as a species, naturally evolve to escape pathogen threats. For instance, it is likely that humans have evolved towards sexual reproduction to increase our range of pathogen-recognition genes by randomly mixing parental genetic material. Given our limited life span, there is little opportunity for pathogens to adapt to this ever-renewed population-level immune system. Furthermore, we have developed knowledge and tools that can help us prevent pandemics, if we learn how to use them collectively. Shah emphasizes the importance of enhancing surveillance globally, as "a kind of global immune system" allowing rapid detection of outbreaks, both in humans and in animals.

What Shah's parallel between cholera and modern pandemics underscores, is that oblivion, a recurring theme of the book, is a critical impediment to optimally responding to pandemics. Nineteenth century New York City faced cholera because of forgotten ancient knowledge of water sanitation. Late twentieth century physicians, who had forgotten Alexander Fleming's warning about resistance to antibiotics, inadequately prescribed them, leading to the emergence of resistant bacterial strains. Arguably, the West African Ebola epidemic was rooted in similar ignorance. The outbreak reached an unprecedented scale because of failure to recognize the virus, although it was well known in other regions; because local populations had forgotten that governments can sometimes act for the common good; and finally because we, the developed world, ignored the issue until it was directly threatening us. Improving our collective memory of past experiences dealing with pathogens is a crucial step to enhance our human response in the race against pathogens. This thoughtprovoking and well-documented book will contribute to spreading the word... I wish it a 'pandemic success'.

\section{REVIEWED BY ANNE CORI}

Anne Cori is a Research Fellow at Imperial College London, Norfolk Place, London W2 1PG, UK.

e-mail:a.cori@imperial.ac.uk 